\title{
CORRELAÇÃO ENTRE A SATISFAÇÃO E EQUILÍBRIO DE INDIVÍDUOS PARKINSONIANOS AO TRATAMENTO EM FORMATO DE CIRCUITO DE TREINAMENTO
}

Katiane Mayara Guerrero, Silas de Oliveira Damasceno, Andressa Sampaio Pereira, Caroline Nunes Gonzaga, Isabela Bortolim Frasson, Alice Haniuda Moliterno, Guilherme Yassuyuki Tacao, Lúcia Martins Barbatto, Augusto Cesinando de Carvalho.

Universidade Estadual Paulista "Júlio Mesquita Filho" - UNESP, Departamento de Fisioterapia, Curso de Fisioterapia, Presidente Prudente, SP. E-mail: katianeguerrero@hotmail.com.

\section{RESUMO}

A avaliação da satisfação de parkinsonianos e sua correlação com o equilíbrio ainda não estão estabelecidos, assim, é fundamental utilizar instrumentos capazes de mensurar tais aspectos. 0 objetivo foi verificar a satisfação de parkinsonianos em tratamento em Fisioterapia em Grupo no Formato de Circuito de Treinamento (FGCT) e correlacionar com o equilíbrio. Foi realizada a avaliação inicial utilizando a Escala Modificada de Berg (EMB) para avaliar o equilíbrio estático e dinâmico e após a intervenção com FGCT, a segunda avaliação acrescentou-se a Escala de Avaliação da Satisfação dos Pacientes Abreviada (SATIS-BR) que analisa a percepção dos pacientes sobre os aspectos dos serviços recebidos, composta por perguntas de caráter quantitativo e qualitativo. A análise estatística não revelou diferença significante na $\operatorname{EMB}(p=0,354)$ e demonstrou uma correlação fraca entre EMB x SATIS-BR ( $r=0,057$ e $p=0,875)$. A FGCT foi capaz de manter o quadro clínico na população analisada.

Palavras-chave: Parkinson, equilíbrio, satisfação ao tratamento, circuito de treinamento, fisioterapia.

\section{CORRELATION BETWEEN THE SATISFACTION AND BALANCE OF INDIVIDUALS WITH PARKINSON'S DISEASE TO TREATMENT IN THE FORM OF A TRAINING CIRCUIT}

\begin{abstract}
The evaluation of parkinsonian satisfaction and its correlation with the balance are still not established, so it is fundamental to use instruments capable of measuring these aspects. The objective was to verify the satisfaction of Parkinsonians in Group Physiotherapy treatment in the Training Circuit Format (FGCT) and correlate with the balance. The initial evaluation was performed using the Berg Modified Scale (EMB) to evaluate the static and dynamic balance and after the intervention with FGCT, the second evaluation was added the Abbreviated Patient Satisfaction Assessment Scale (SATIS-BR), which analyzes the patients' perception about the aspects of the services received, consisting of quantitative and qualitative questions. Statistical analysis revealed no significant difference in BMS $(p=0.354)$ and showed a weak correlation between BMS $\times$ SATIS-BR $(r=0.057$ and $p=0.875)$. The FGCT was able to maintain the clinical picture in the analyzed population.
\end{abstract}

Keywords: Parkinson, balance, treatment satisfaction, training circuit, physiotherapy. 


\section{INTRODUÇÃO}

A doença de Parkinson ocasiona alterações sistêmicas que influenciam diretamente na funcionalidade do paciente, tendo como principais características, o tremor, a bradicinesia e a rigidez que podem levar a falta de equilíbrio ${ }^{1}$.

Dentro da gama de sinais e sintomas, o equilíbrio se destaca por ser um processo complexo e que necessita da integração do sistema vestibular e periférico, do sistema visual e dos sistemas neuromusculares e centrais para que o indivíduo consiga manter o seu centro de gravidade dentro de um limite estável ${ }^{1}$.

Por isso, o tratamento fisioterapêutico deve apresentar uma abordagem que impacta nos domínios sociais, biológicos e psicológicos dos pacientes neurológicos, pois estes aspectos influenciam na prática clínica ${ }^{2}$. Nesta vertente, avaliar a satisfação do paciente frente ao próprio tratamento, se torna uma ferramenta fundamental na delimitação de uma intervenção por parte dos profissionais envolvidos nos cuidados ao paciente ${ }^{3}$.

Por definição, a satisfação é a percepção do indivíduo frente a uma expectativa prévia sobre o tratamento com base nas experiências vividas comparada com a observação do atual cenário em que esta inserido, com isso, o avaliado consegue apontar a qualidade do serviço em que esta em tratamento, aspectos terapêuticos que necessitam de ajustes e dentre outros ${ }^{4}$.

Portanto, tornar o paciente parte do processo de construção do seu próprio tratamento faz com que o mesmo se torne o principal agente de sua evolução terapêutica, uma vez que, analisar os vislumbres, os desejos e as inquietações dos pacientes em tratamento mostra-se como um artifício estratégico de valorização pessoal ${ }^{5}$.

Logo, utilizar de instrumentos que sejam capazes de mensurar a satisfação e o equilíbrio de parkinsonianos são fundamentais, tendo dentro do espectro de avaliações da satisfação destaque para a Escala de Satisfação dos Pacientes com os Serviços de Saúde Mental (SATIS-BR) ${ }^{6}$, assim como, para o equilíbrio a Escala Modificada de Berg $^{7}$ que podem se tornar parâmetros para futuras intervenções em parkinsonianos.

Em consonância, dentro dos diversos tipos de tratamentos existentes, a Fisioterapia em Grupo no Formato de Circuito de Treinamento (FGCT) tem se mostrado como modelo terapêutico viável, na qual se utiliza de estações interligadas que simulam atividades do cotidiano e tem apresentado benefícios na melhora da independência funcional e na manutenção de habilidades motoras dos participantes ${ }^{8}$.

No entanto, apesar da doença de Parkinson ser amplamente estuda, ainda não se sabe com clareza, se existe uma correlação entre a satisfação ao tratamento com o equilíbrio desta população, por isso, a necessidade de tal avaliação, uma vez que estas informações podem se tornar dados modeladores para um bom tratamento. Assim, o atual estudo tem por objetivo verificar a satisfação de parkinsonianos ao tratamento de FGCT e correlacionar com o equilíbrio.

\section{METODOLOGIA}

Para realização deste estudo clínico foram recrutados 10 parkinsonianos atendidos em um centro de de Fisioterapia e Reabilitação na Universidade Estadual Paulista "Júlio de Mesquita Filho" (UNESP), campus de Presidente Prudente-SP.

Foram incluídos neste estudo pacientes de ambos os sexos, com diagnostico clínico de Doença de Parkinson, Hoehn e Yahr 1, 2 e 3, ausência de déficits cognitivos avaliados pelo miniexame do estado mental e que aceitaram assinar o Termo de Consentimento Livre e Esclarecido aprovado pelo Comitê de Ética em Pesquisa da FCT/UNESP e cujos procedimentos adotados obedecem aos Critérios da Ética em Pesquisa com Seres Humanos conforme Resolução no. 466/2012 do Conselho Nacional de Saúde (CAAE 80229017.0.0000.5402).

Antes de iniciar o treinamento de FGCT, os pacientes foram submetidos a uma entrevista individual para coleta de dados sociodemograficos e logo após foi realizada a avaliação inicial 
(AV1) utilizando a Escala Modificada de Berg. Após 8 semanas de intervenção com FGCT, foi realizada uma segunda avaliação (AV2) utilizando-se a mesma escala de avaliação e a escala de satisfação do tratamento.

\section{Escala modificada de berg}

A Escala Modificada de Berg (EMB) é capaz de discriminar idosos propensos à queda e é muito utilizada em avaliações clinicas para testar o equilíbrio em parkinsonianos e consiste em tarefas de equilíbrio sobre um dos hemicorpos, de transferências, em posturas dinâmicas e estáticas. Os itens avaliados recebem uma pontuação de 0 a 4 baseados na habilidade para atingir o tempo específico e a distância requeridos no teste. Uma pontuação '0' representa inabilidade para completar um item e uma pontuação '4' representa a habilidade de completar independentemente a tarefa. O teste totaliza 56 pontos e abaixo de 36 pontos o risco de quedas é de $100 \%$, ao passo que, com o aumento dos pontos o risco de queda é cada vez menor ${ }^{7}$.

\section{Escala de avaliação da satisfação dos pacientes abreviada (satis-br)}

O questionário na versão breve constitui-se de 15 questões que incluem perguntas de caráter quantitativo da escala SATIS-BR e qualitativo, relacionadas à percepção dos pacientes sobre diferentes aspectos dos serviços recebidos e aos seus dados sociodemográficos. Propõe avaliar o grau de satisfação dos pacientes com relação ao entendimento dos terapeutas sobre seu problema, o grau de satisfação correspondente à ajuda recebida no serviço e o grau de satisfação ao estado físico e conforto do tratamento.

A escala SATIS-BR propriamente dita é composta por 12 itens que calculam o grau de satisfação dos pacientes com os atendimentos prestados. Estes itens contêm opções de resposta em uma escala tipo Likert (que varia de um extremo ao outro, ou seja, de muito insatisfeito (1 ponto) até muito satisfeito (5 pontos). Portanto, quanto maior for a média dos escores obtidos na aplicação da escala maior será o grau de satisfação. Além disso, possui três questões abertas qualitativas que avaliam os aspectos do serviço mais e menos apreciados pelo paciente, bem como suas sugestões para melhorias ${ }^{9,10}$.

\section{Fisioterapia em grupo no formato de circuito de treinamento}

A FGCT aconteceu com 14 áreas diferentes, de modo que em cada uma há um distinto grau de dificuldade de realização do exercício. As estações do circuito foram montadas no CEAFIR. Foram utilizados bastões, steps, halteres, caneleiras, escada progressiva, rampa, barras paralelas, garrafas, elásticos, cones, dentre outros objetos. Inicialmente é realizada uma dança terapêutica, na qual envolve movimentos corporais globais e logo após se inicia o circuito com as seguintes estações: 
Tabela 1. descrição dos exercícios executados nas estações

Estação 1 Marcha com dupla tarefa, o parkinsoniano deve falar nome de frutas com a letra determinada pelo terapeuta enquanto anda.

\begin{tabular}{|c|c|}
\hline Estação 2 & Bicicleta ergométrica \\
\hline Estação 3 & $\begin{array}{l}\text { Condicionamento respiratório - o parkinsoniano deve assoprar uma bola de } \\
\text { isopor para o outro lado da caixa }\end{array}$ \\
\hline Estação 4 & $\begin{array}{l}\text { O parkinsoniano deve dar passo lateral, para frente e para trás dentro de um } \\
\text { quadrado desenhado no chão }\end{array}$ \\
\hline Estação 5 & $\begin{array}{l}\text { Rotação de tronco - Transferência de garrafas plásticas, com } 1 \text { quilograma, de } \\
\text { uma mesa para um degrau colocado ao lado no chão e vice versa. }\end{array}$ \\
\hline Estação 6 & $\begin{array}{l}\text { Lemocot adaptado: individuo sentado na cadeira com dois pontos paralelos a sua } \\
\text { frente, o mesmo deve tocar com a ponta do pé um ponto de cada vez. }\end{array}$ \\
\hline Estação 7 & Movimento de agachamento com step \\
\hline Estação 8 & $\begin{array}{l}\text { Marcha - Caminhar numa distância de } 5 \text { metros em relação a um cone enquanto } \\
\text { faz rotação com uma bolinha em volta do corpo, retornar à posição e repetir o } \\
\text { procedimento }\end{array}$ \\
\hline Estação 9 & $\begin{array}{l}\text { Motricidade fina - colocar e retirar prendedores de um barbante preso em uma } \\
\text { prateleira }\end{array}$ \\
\hline Estação 10 & $\begin{array}{l}\text { Marcha na barra paralela com obstáculos de } 5 \mathrm{~cm} \text { de altura distantes entre si por } \\
30 \mathrm{~cm} \text {. }\end{array}$ \\
\hline Estação 11 & $\begin{array}{l}\text { Bambolê - com o indivíduo colocando-se dentro do bambolê, o mesmo deve } \\
\text { leva-lo ao chão e levanta-lo acima de sua cabeça }\end{array}$ \\
\hline Estação 12 & Subir numa escada, a seguir descer numa rampa e vice versa. \\
\hline Estação 13 & $\begin{array}{l}\text { Movimento de flexão de ombro - } 2 \text { alvos na parede, colocados a 1,50 metros do } \\
\text { chão, com uma distância de } 1 \text { metro em relação ao outro }\end{array}$ \\
\hline Estação 14 & Flexão de tronco utilizando um bastão \\
\hline
\end{tabular}

\section{Análise estatística}

Os dados foram tabulados em planilhas pré-definidas no software Microsoft Office Excel e testados quanto à distribuição normal pelo teste de Shapiro wilk. As estatísticas descritivas (média e desvio padrão) foram operacionalizadas pelo Statistical Software for Social Sciences (SPSS Inc. Chicago, IL) versão 18.0, bem como o teste de normalidade, comparações e análises de correlação. $O$ teste $t$ de student para amostras pareadas foi utilizado para comparar os grupos. O coeficiente de correlação usado foi Correlação de Pearson ( $r$ ) para dados com distribuição normal. Para todas as análises foi considerado nível de significância de $\alpha=0,05$.

Para as diferenças entre as avaliações também foi calculado o Effect Size (ES), pela fórmula Cohen (d). As leituras das magnitudes foram realizadas como efeito insignificante $(>=0,00$ a 0,15$)$; pequeno efeito $(>=0,15$ e $<0,40)$; médio efeito $(>=0,40$ e $<0,75)$; grande efeito $(>0,75)$. Esse conceito estatístico é traduzido normalmente pela diferença efetiva na população, sendo assim, quanto maior for o ES, maior será a manifestação do fenômeno na população ${ }^{11}$.

\section{RESULTADOS}

Participaram desse estudo 10 parkinsonianos, sendo 9 homens e 1 mulher, a amostra analisada apresentou idade em média de 61,39 \pm 6,38 anos. Os resultados da EMB na AV1 
demonstraram $54,3 \pm 1,82$ pontos e $55,0 \pm 1,24$ na $A V 2$, todavia a análise estatística não revelou diferença significante entre as avaliações $(p=0,354)$, já o SATIS-BR mostrou uma pontuação de 4,69 $\pm 0,29$ pontos. O teste de Pearson demonstrou uma correlação fraca entre EMB $x$ satisfação ao tratamento $(r=0,057$ e $p=0,875)$ e o effect size demonstrou médio efeito para $\operatorname{EMB}(d=0,47)$.

\section{DISCUSSÃO}

O presente estudo evidenciou que a correlação entre a satisfação dos pacientes com o tratamento fisioterapêutico e a EMB não houve diferença estatística significante, bem como, também não apresentou diferença estatística entre AV1 a AV2 na EMB. Entretanto, quando observado o effect size confirmou um médio efeito para EMB, estes achados podem ser justificados devido aos valores tanto inicias como finais se manterem classificados na escala como um bom equilíbrio, no qual esta varia entre um escore de 41 a 56 pontos ${ }^{12,13}$. Logo, esses pacientes mantiveram seu estado clinico, ou seja, não pioraram seu quadro, o que sugere que a proposta de tratamento com FGCT é positiva.

Apesar de a FGCT ser pouco relatada nessa população, estudos recentes já realizados demonstram efeitos benéficos ${ }^{14,15}$, corroborando com os achados do presente estudo, visto que esse tipo de intervenção tem como proposta auxiliar na melhora do estado de saúde global, por meio de um ambiente capaz de manter a socialização entre os indivíduos, além de proporcionar uma aprendizagem motora e a realização de tarefas para a manutenção do equilíbrio e funcionalidade dos pacientes ${ }^{14}$, tornando-se uma intervenção promissora para manter esses indivíduos ativos.

O presente estudo identificou a necessidade de mais pesquisas sobre o assunto, visto que apesar de ter modelos de pesquisas relacionados ${ }^{15,16}$, estes possuem objetivos e instrumentos diferentes ${ }^{15,17,18,19}$, demonstrando uma escassez de estudos na literatura principalmente sobre a escala SATIS-BR nessa população, bem como no modelo de intervenção. Pode-se ainda reforçar a relevância de programas terapêuticos alternativos e dinâmicos que busquem o aumento da atividade física e o cuidado psicoterapêutico, visto que estes podem estar correlacionados com processos neurofisiológicos positivos ${ }^{15}$.

Contudo, o estudo apresenta limitações nos quais se referem ao número reduzido da amostra, classificações diferentes pelo Hoehn e Yahr, comprometimento motor heterogêneo e diferentes idades, que somados, podem ter influenciado nos resultados apresentados. Estudos futuros que seguirem o mesmo delineamento metodológico deverão levar em consideração os pontos aqui apresentados para obtenção de resultados mais precisos.

\section{CONCLUSÃO}

Nota-se que a FGCT apesar de não promover efeito significante entre as avaliações da EMB ou correlação fraca com a satisfação ao tratamento, houve um médio efeito no effect size e conseguiu aumentar e/ou manter a pontuação na escala, o que denota que o tratamento manteve o quadro clínico na população analisada sem ocorrer declínio funcional.

\section{REFERÊNCIAS}

1. Maia AC, Rodrigues PF, Magalhães LC, Teixeira RLL. Crosscultural adaptation and analysis of the psychometric properties of the Balance Evaluation Systems Test and MiniBESTest in the elderly and individuals with Parkinson's disease: application of the Rasch model. Braz J Phys Ther. 2013;17(3):195-217. DOI: dx.doi.org/10.1590/ S1413-35552012005000085

2. Oliveira AIC, Silveira KRM. Utilização da CIF em pacientes com sequelas de AVC. Ver. Neurocienc. 2011; 19(4):653-662. 
3. Fontes, MSV, Collares PMC. Satisfação de usuário no serviço de fisioterapia em clínicas de fortaleza. Rev. Cientefico. 2004; 14(29):23-35.

4. Bandeira M, Silva MA. Escala de Satisfação dos Pacientes com os Serviços de Saúde Mental (SATIS-BR): estudo de validação. J Bras Psiquiatr, 2012; 61(3):124-32. http://dx.doi.org/10.1590/S0047-20852012000300002.

5. Kantorski LP, Jardim VMR, Treichel CAS, Demarco DA, Neutzling AS, Oliveira MM, et al . Satisfaction with mental health community services among patients' relatives. Rev. bras. epidemiol., 2017; 20(2): 237-246. DOI: http://dx.doi.org/10.1590/1980-5497201700020005.

6. Bandeira M, Silva MA, Aparecida CC, Mara FC. Satisfação de familiares de pacientes psiquiátricos com os serviços de saúde mental e seus fatores associados. J. bras. Psiq., 2011; 60(4): 284-293. DOI: http://dx.doi.org/10.1590/S0047-20852011000400009.

7. Berg K, Wood-Dauphinee S, Williams JI. The Balance Scale - Reliability Assessment with Elderly Residents and Patients with an Acute Stroke. Scandinavian Journal of Rehabilitation Medicine. 1995; 27(1): 27-36.

8. Carvalho AC, Pereira AS, Oliveira MCS, Franco MR. Fisioterapia em grupo no formato de circuito de treinamento pós-acidente vascular cerebral. In: Associação Brasileira de Fisioterapia Neurofuncional; Garcia CSNB, Facchinetti LD, organizadoras. PROFISIO Programa de Atualização em Fisioterapia Neurofuncional: Ciclo 5. Porto Alegre: Artmed Panamericana; 2018; 3:41-75.

9. Da Silva MA, Bandeira M, Scalon JD, Quaglia MAC. Satisfação dos pacientes com os serviços de saúde mental: a percepção de mudanças como preditora. Jornal Brasileiro Psiquiatria. 2012; 61(2):64-71.https://doi.org/10.1590/S0047-20852012000200002

10. Monnin, Dominique; Thomas V. Perneger. Scale to measure patient satisfaction with physical therapy. Physical therapy. 2002; 82(7):682-691.

11. Loureiro LMJ, Gameiro MGH. Interpretação crítica dos resultados estatísticos: para lá da significância estatística. Rev Enferm Ref. 2011; 3(3):151-162. https://doi.org/10.12707/RIII1009

12. Miyamoto ST, Junior LJ, Berg KO, Ramos, L.R, Natour J. Brazilian version of the Berg balance scale. Brazilian Journal of Medical and Biological Research. 2004; 37(9):1411-1421. DOI: http://dx.doi.org/10.1590/S0100-879X2004000900017.

13. Kuriki HU; Carvalho AC, Bofi TC, Pereira MB. Déficit de equilíbrio e agilidade em indivíduos com doença de Parkinson e sua correlação com a independência funcional da marcha. Geriatria \& Gerontologia. 2011; 5(2):86-90.

14. Pereira JAC, Gaiad TP, Santos AP. Efeito do treinamento em circuito orientado para tarefa sobre o desempenho motor e a qualidade de vida de pacientes com doença de Parkinson: um estudo de casos. Revista de Terapia Ocupacional da Universidade de São Paulo. 2016; 27(3):32935. DOI: https://doi.org/10.11606/issn.2238-6149.v27i3p329-335.

15. Choudhury TK, Harris C, Crist K, Satterwhite TK, York MK. Comparative Patient Satisfaction and Feasibility of a Pilot Parkinson's Disease Enrichment Program. Journal Of Geriatric Psychiatry And Neurology. 2017; 30 (5):253-260. DOI: doi: http://dx.doi.org/10.1177/0891988717720299. 
16. Straudi S, Martinuzzi C, Pavarelli C, Sabbagh Charabati A, Benedetti MG, Foti C, et al. A taskoriented circuit training in multiple sclerosis: a feasibility study. BMC Neurol. 2014; 7(14):1-9. DOI: https://doi.org/10.1186/1471-2377-14-124.

17. Keus SH, Bloem BR, Verbaan D, de Jonge PA, Hofman $M$, van Hilten BJ, et al. Physiotherapy in Parkinson's disease: utilisation and patient satisfaction. Journal Of Neurology. 2004; 251(6):68087. DOI: https://doi.org/ 10.1007/s00415-004-0402-7.

18. Wilkinson JR, Spindler M, Wood SM, Marcus SC, Weintraub D, Morley JF et al. High patient satisfaction with telehealth in Parkinson disease. Neurology: Clinical Practice. 2016; 6(3):241-251. DOI: https://doi.org/10.1212/CPJ.0000000000000252.

19. Gal O, Srp M, Konvalinkova R, Hoskovcova M, Capek V, Roth J, Ruzicka E. Physiotherapy in Parkinson's Disease: Building ParkinsonNet in Czechia. Parkinson's Disease. 2017; 2017:1-7. DOI: https://doi.org/10.1155/2017/8921932. 